\title{
Efficacy and Safety in Intramuscular Injection Techniques Using Ultrasonographic Data
}

\author{
Tetsuya Tanioka ${ }^{1}$, Kensaku Takase ${ }^{2}$, Yuko Yasuhara1, Yueren Zhao ${ }^{3}$, Chizuru Noda ${ }^{4}$, \\ Saki Hisashige ${ }^{4}$, Rozzano Locsin ${ }^{1}$
}

\author{
${ }^{1}$ Department of Nursing Institute of Biomedical Sciences, Tokushima University Graduate School, Tokushima, Japan \\ ${ }^{2}$ Department of Rehabilitation, Tokushima Prefectural Central Hospital, Tokushima, Japan \\ ${ }^{3}$ Department of Neuropsychiatry, Fujita Health University, Toyoake, Japan \\ ${ }^{4}$ School of Health Sciences, Tokushima University, Tokushima, Japan \\ Email: tetsuyayt@gmail.com
}

How to cite this paper: Tanioka, T., Takase, K., Yasuhara, Y., Zhao, Y.R., Noda, C., Hisashige, S. and Locsin, R. (2018) Efficacy and Safety in Intramuscular Injection Techniques Using Ultrasonographic Data. Health, 10, 334-350.

https://doi.org/10.4236/health.2018.103027

Received: February 1, 2018

Accepted: March 24, 2018

Published: March 27, 2018

Copyright (C) 2018 by authors and Scientific Research Publishing Inc. This work is licensed under the Creative Commons Attribution International License (CC BY 4.0).

http://creativecommons.org/licenses/by/4.0/

\begin{abstract}
Delivered by intramuscular (IM) injections, Long Acting Injections (LAI), offering comparatively long term medicinal effects from several weeks to several months, are gaining much attention. The aim of this article was to indicate a safe and effective IM injection technique based on evidenced data derived from ultrasonographic data. Accurate anatomical knowledge is necessary in order to administer IM injections. It is possible to gain an adequate understanding of injection site anatomical structures by using ultrasound diagnostic devices. In particular, ultrasonography offers real-time diagnostic data allowing for observation of injection site, subcutaneous tissues, fat layers, muscle membrane, muscles and bones. To deliver the drug accurately into the muscle is essential, to achieve maximum drug efficacy and prevent injection site reactions. Therefore, when administering IM injections to the buttocks area, a suitable injection needle between $23 \mathrm{G} 25 \mathrm{~mm}$ to $21 \mathrm{G}$ or $22 \mathrm{G} 38 \mathrm{~mm}$ must be chosen depending on physique and drug characteristics. Needles determined as safe and effective through ultrasonographic evidence are needed such as in the case of a deltoid injection site, in which a $23 \mathrm{G} 25 \mathrm{~mm}$ injection needle is used in the absence of notable obesity. Not only the needle type is essential, but also the IM procedure itself. It is important to stretch the skin, inserting the needle at 90 degree angle to ensure depth of needle insertion to the muscle. Until new confirmed evidence becomes available, it is necessary to increase the understanding of injection site selection methods, needle insertion depth, needle angle, and established IM injection techniques. The application of ultrasound devices in the development of next-generation techniques for IM injection is promising. Importantly, these techniques provide critical information from a risk management perspective.
\end{abstract}




\section{Keywords}

Intramuscular Injection, Long Acting Injectable, Injection Site Reaction, Ultrasound Diagnostic Device, Intramuscular Injection Technique

\section{Introduction}

Delivered by intramuscular (IM) injections, Long Acting Injections (LAI), offering comparatively long term medicinal effects from several weeks to several months, are gaining much attention [1] [2] [3] [4]. However, there are dangers such as vascular damage, muscle contracture, nerve injury due to IM injection [5] [6] [7] [8] [9], and injections site reactions such as redness and swelling, which may occur [10] [11]. In particular, oily formulation LAIs, which have been designed so that the drug metabolizes in the muscle, contain aspects warranting careful attention, such as being more irritant than the usual medicines [12], and having a higher viscosity. Moreover, in administration of injectable medications, physicians and nurses are unable to confirm visually whether or not the tip of the injection needle has reached the muscle. Therefore, physicians and nurses need to select an appropriate injection needle penetration depth in conjunction with patient physique [13], injection site [14] and anatomical understanding of nerves and blood vessels.

In consideration of safety aspects for the IM injection such as the prevention of nerve and blood vessel injury, and injection site reactions such as redness and swelling, 1) selection of appropriate IM injection sites (See Section 2), 2) selection of appropriate IM injection needles (See Section 3), 3) assessment of IM injection site by ultrasound diagnostic device (See Section 4), and 4) points to acquire IM injection technique (See Section 5) are key issues for physicians and nurses to ensure that the drugs are dispensed into the muscle [15].

The method of choosing an appropriate IM injection site on the body involves utilizing traditional methods, in deciding the injection site, penetration point of the injection needle and subsequently piercing the skin vertically to penetrate the muscle. In IM injections currently and commonly being administered, the penetration point is the only aspect being decided. However, it is crucial that the needle injecting the medicine is administered in the optimum direction and length. In reality, this is likely reliant upon the experience and intuition of the physician and nurses [16]. As such, the current IM injection method has its limitations, featuring a multitude of problems in terms of ensuring medical safety and establishing a proven IM injection technique. The authors' research team has experience in the use of ultrasound diagnostic devices to conduct analysis of drug luminance changes post-IM injection [17], the relationship between injection site reactions and treatment efficacy according to IM injection needle penetration depth [11], and research involving appropriate and safe needle penetration length in IM injections [15] [18] [19] [20]. Data were obtained from 136 patients undergoing treatment with LAI and 83 healthy subjects by ultrasonogra- 
phy at four psychiatric hospitals and one psychiatric clinic in Japan.

The aim of this article was to indicate a safe and an effective IM injection technique based on evidenced data derived from ultrasonographic data.

\section{Selection of Appropriate Injection Site}

\subsection{Appropriate Assessment of the Gluteus Muscle IM Injection Sites}

Conventionally, when carrying out IM injections, areas featuring more highly developed muscles are chosen, and certainly to inject into the muscle has been considered crucial. Possible sites for the adult IM injection include: deltoid, dorsogluteal, and ventrogluteal muscles (Figure 1(a), and Figure 1(b)) [21].

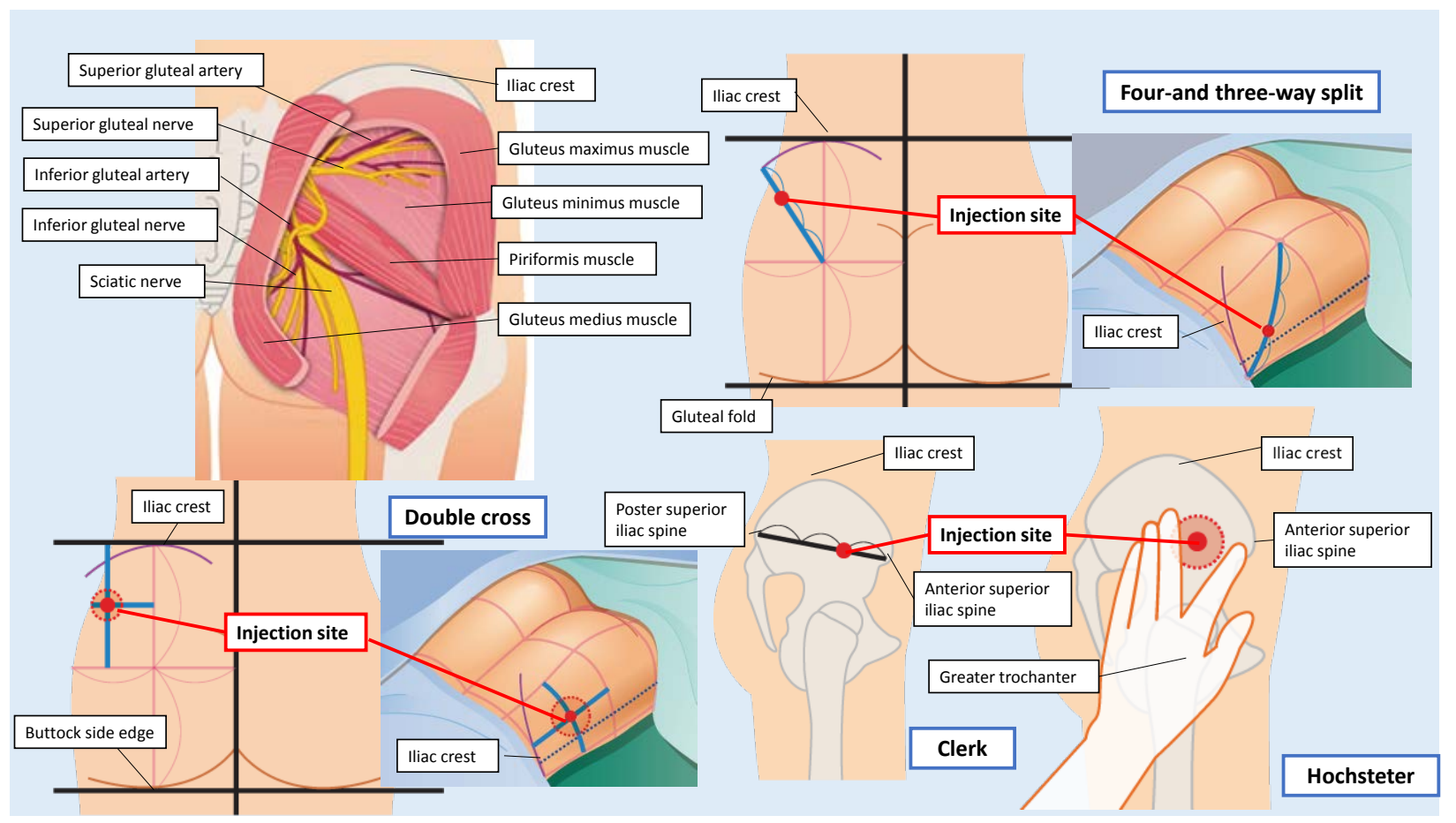

(a)

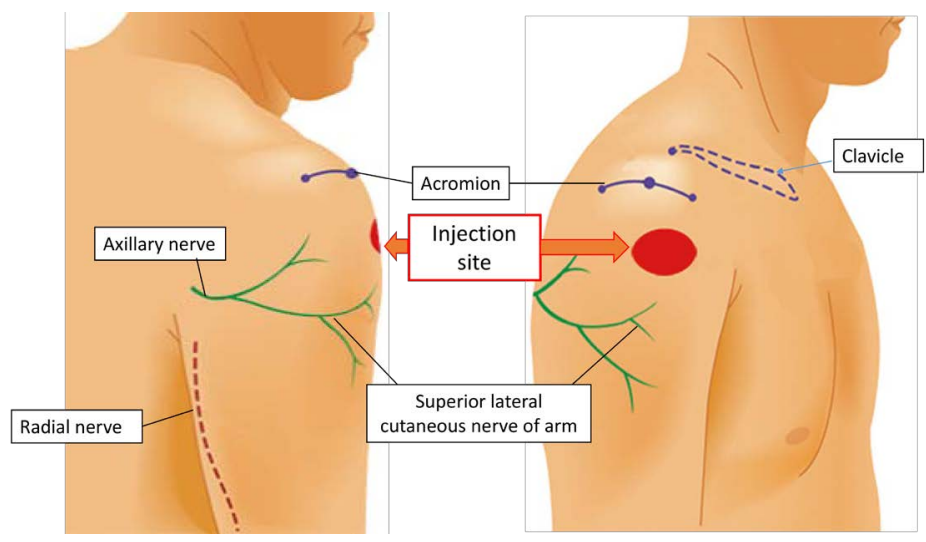

(b)

Figure 1. (a) Gluteal IM injection sites; (b) Deltoid IM injection site. 
In clinical practice in Japan, the principal methods for assessing appropriate IM injection sites of the buttocks are the dorsogluteal four and three-way split method [22] and double cross method [23] [24]. However, since the four and three-way split method point involves an area proximal to where the superior gluteal artery has many branches in the buttocks, particular care must be taken to avoid not only nerve damage, but also blood vessel puncture [22]. The double-cross method [23] [24] is a method which was proposed to avoid the risk of nerve injury to the sciatic nerve [25] [26]. However, it is close to the four-three way split method point, so caution is required for the aforementioned reasons. In addition, there are also the Hochstetter and Clark points located on the ventrogluteal site [27]. All these methods are specialized methods for the IM injection regions proposed in the 1960s [26], and no revolutionary injection site selection methods have been proposed since. These injection site assessment methods are a guide to IM injection penetration points, at which it should be theoretically and experientially difficult to damage nerves or blood vessels if one were to administer an injection at these points.

It is however necessary to be mindful of the fact that given individual differences in physique and pelvis shape [28], inappropriate penetration direction or depth can cause problems.

\subsection{Assessment of Deltoid Muscle IM Injection Site}

The three finger breadths below the acromion is selected as the site for the deltoid IM injection site. Although opinion is divided, the shortest distance from the acromion to the axillary nerve is said to be $5 \mathrm{~cm}$. Given the close proximity of the deltoid IM injection site to nerves and blood vessels, it is especially important to correctly understand anatomical physiology and be mindful of safety. There are examples of the deltoid branch of the axillary muscle and blood vessels running through the thickest section, previously determined by inspection and palpation to have sufficient muscle [22]. Moreover, there are also reports of vaccination-related shoulder dysfunction due to injection into the upper third of the deltoid muscle [29]. It is crucial to accurately determine the position of the acromion which serves as an indicator in ascertaining the safe area of the deltoid muscle, while paying heed to patients' individual differences (sex, physique, body-mass-index etc.) [30].

Therefore, it is becoming increasingly important for clinicians to identify a safer IM injection site in the deltoid muscle because of possible complications following the vaccine administration of IM injections [31].

\section{Selection of Injection Needle, Insertion Depth of Injection Needle}

\subsection{Selection of Injection Needle, Insertion Depth of Injection Needle in Gluteal IM Injection Sites}

In order to administer the drug into the gluteus medius muscle, the entire injec- 
tion needle tip needs to reach the sub fascial, and it is important to assess the distance to the targeted gluteus medius muscle [32].

Using an ultrasound diagnostic device to measure the thickness of subcutaneous fat, Kikuchi et al. [33] reported figures for subjects under 65 years of age, at the four and three-way point, such as males: $1.05 \pm 0.43 \mathrm{~cm}$ and females: 1.41 $\pm 0.48 \mathrm{~cm}$.

From the results of their cadaver dissections, Sato et al. [34] reported that for 11 out of 32 cases the gluteus maximus muscle was distributed at the four and three-way point. For subjects whose gluteus maximus muscle was found to overlay the gluteus medius muscle, the distance to the gluteus medius muscle from the skin was $3.8 \pm 2.3 \mathrm{~cm}$, however, in patients without the distributed gluteus maximus muscle, the distance was $1.6 \pm 0.8 \mathrm{~cm}$. Thus, it is necessary to determine an injection needle depth with respect to the presence or absence of gluteus maximus muscle distribution, if one intends the needle point to reach the gluteus medius muscle at this injection site.

The distance to the gluteus medius muscle is determined by two factors; the thickness of subcutaneous fat directly beneath the injection site, and the presence or absence of the gluteus medius muscle (whether the gluteus maximus is atop the gluteus medius or not). Notwithstanding, results of dorsogluteal measurements for Japanese subjects are available. Given that physique differs according to ethnicity, there is a need to refer to national averages for physique, when regarding survey results.

\subsection{Selection of Appropriate IM Injection Needle}

While there are various types of injection needles available, in the case of IM injection, 21 - 23G injection needles are used, and for oil-based formulations 21G, $1.1 / 2(38 \mathrm{~mm}) \mathrm{RB}$ (regular bevel) is preferable.

Nevertheless, the majority of nurses working in the psychiatric department used the 23G, RB 1.1/4 injection needle (32 mm) [35]. When using a 23G, RB $1.1 / 4$ injection needle $(32 \mathrm{~mm})$ to administer an IM injection at the four and three-way split method site, even if the needle were inserted to the base, there is a possibility that it may not reach the gluteus medium muscle, and the drug may be injected into the subcutaneous or gluteus maximus muscle [36] [37]. Therefore, it is thought that when selecting a four three-way split method site, and injecting the buttocks presenting with thick subcutaneous fat, it may be necessary to employ long 22G1.1/2 (38 mm) needles [38].

In some cases, if the insertion depth is shallow than as expected, IM injection needle may not penetrate the muscle fascia. However, if needle insertion depth is deeper than anticipated, needle penetration may cause damage to nerves, arteries and veins. This provided the authors the impetus to evaluate the suitable depth of injection needles [11]. The plasma 9-hydroxyrisperidone (9-OH-RIS) concentrations were measured every two weeks. Comparison data were determined on the 8 th week and the 14 th week. No significant difference was observed in 
9-OH-RIS concentrations, psychotic symptoms, of subjects in both groups. Also, with the $50 \mathrm{~mm}$ needle insertion group, injection site adverse reactions were confirmed in two subjects (15\%). Also, in the $20 \mathrm{~mm}$ needle insertion group, injection site reactions were confirmed in six subjects (46\%). Although effective 9-OH-RIS concentrations were obtained with the insertion site using both depths, it was found that the $50 \mathrm{~mm}$ insertion length was more suitable for dorsogluteal IM injections in adult patients with schizophrenia as demonstrated by the incidence of local adverse skin reactions.

\subsection{Relationship with Body Mass Index (BMI), Muscle Mass, and Body Fat Percentage}

As shown in Figure 2, there are individual differences in muscle mass volume and body fat percentage. In particular, this point needs to be taken into consideration when doing IM injection.

The patients' daily activity levels vary, with some engaged in employment and some not. Furthermore, while there are reports of obesity trends due to increased appetite and reduced motivation side-effects of the anti-psychotic medication [39], there are also reports of weight loss due to reduced appetite in conjunction with mental and physical symptoms (swallowing disorders, masticatory disorders) [40].

There is a possibility of differences in body fat and muscle volume for healthy women using LAI for infertility treatments or contraceptives, and patients undergoing sustained treatment for schizophrenia.

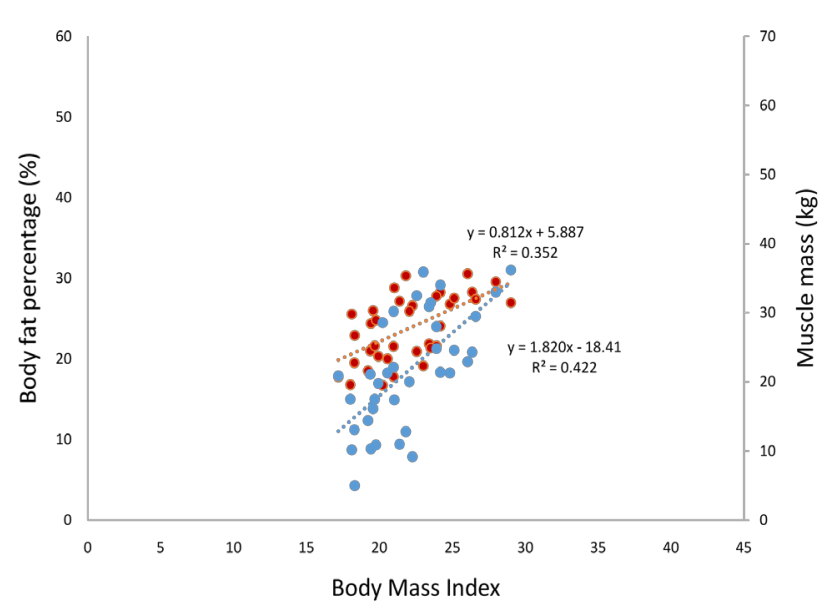

$\mathrm{N}=39$

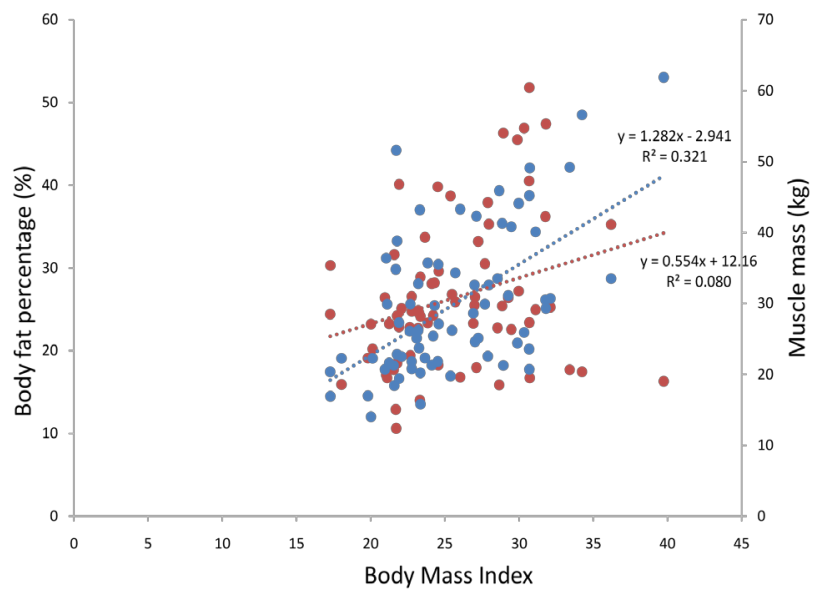

$\mathrm{N}=71$

Muscle mass

Body fat percentage

Figure 2. Relationship with body mass index, muscle mass, body fat percentage: Comparison of healthy subject group (figure, left side), patient with schizophrenia group (figure, right side). In the left scattergram the data of healthy individuals within the body mass index guidelines are exhibited. There is little variation between muscle volume and fat ratio (An unpublished research data by the authors). Meanwhile, the right scattergram presents data from patients undergoing treatment with injections of antipsychotic drugs long-term, due to schizophrenia. 


\section{Assessment of IM Injection Site by Ultrasound Diagnostic Device}

As previously mentioned, accurate anatomical knowledge is necessary in order to administer IM injections. It is possible to gain an adequate understanding of injection site anatomical structures by using ultrasound diagnostic devices. The application of ultrasound diagnostic devices in the development of next-generation IM injection techniques is promising. In particular, ultrasonography offers real-time diagnostic capabilities, allowing for the observation of injection site skin, subcutaneous tissues, fat layers, blood vessels, nerves, fascia, muscle and even muscle fiber. Using the ultrasound image, it is possible to confirm the anatomical structure of the injection site and administer the injection avoiding vessels and nerves. Moreover, it is possible to confirm the direction and depth of the needle, as well as observe the conditions of the surrounding area when the drug is actually administered, in real-time.

\subsection{Ultrasound Diagnostic Image at Dorsogluteal Site}

Figure 3 shows an ulutorasonographic image at dorsoglutal site, visible from the top, skin surface, subcutaneous tissue, fascia, gluteus muscles and ilium.

\subsection{Ultrasonic Diagnostic Image of Deltoid IM Injection Site}

Figure 4 shows an image by ultrasound diagnostic device of the deltoid IM injection site (Right side). Visible from the top are the epidermis, subcutaneous tissue, muscle membrane, muscle and humerus.

\subsection{Aripiprazole Long-Acting Injectable Antipsychotics B-Mode Image: Injection Needle, Fascia, Muscle, Injection Solution, Acoustic Shadow during Injection}

Figures 5(a)-(f) is the ultrasonographic findings during IM injection of the aripiprazole long-acting injectable antipsychotics (ALAI). The ALAI is a water-soluble suspension. It is drawn as high echogenicity on the B-mode scan. In the ultrasound image, the acoustic shadow is noted as a high range brightness toward the back. The injection needle itself casts a strong characteristically metallic acoustic shadow, so it is not possible to directly confirm the injection needle as an image, only the tip of the needle can be monitored, though its movement can be confirmed on video.

\subsection{Detection of Arteries by B-Mode Scan with Colored Doppler Image}

Figure 6 shows a pulsative blood flow (artery) that was detected by B-mode scan with colored Doppler image.

On duplex scan (B-mode scan with colored Doppler), arteries and veins can be detected. The lumen with colored Doppler signals suggests arteries or veins. In this case, arterial pulsatile Doppler signals were detected on duplex scan. The 


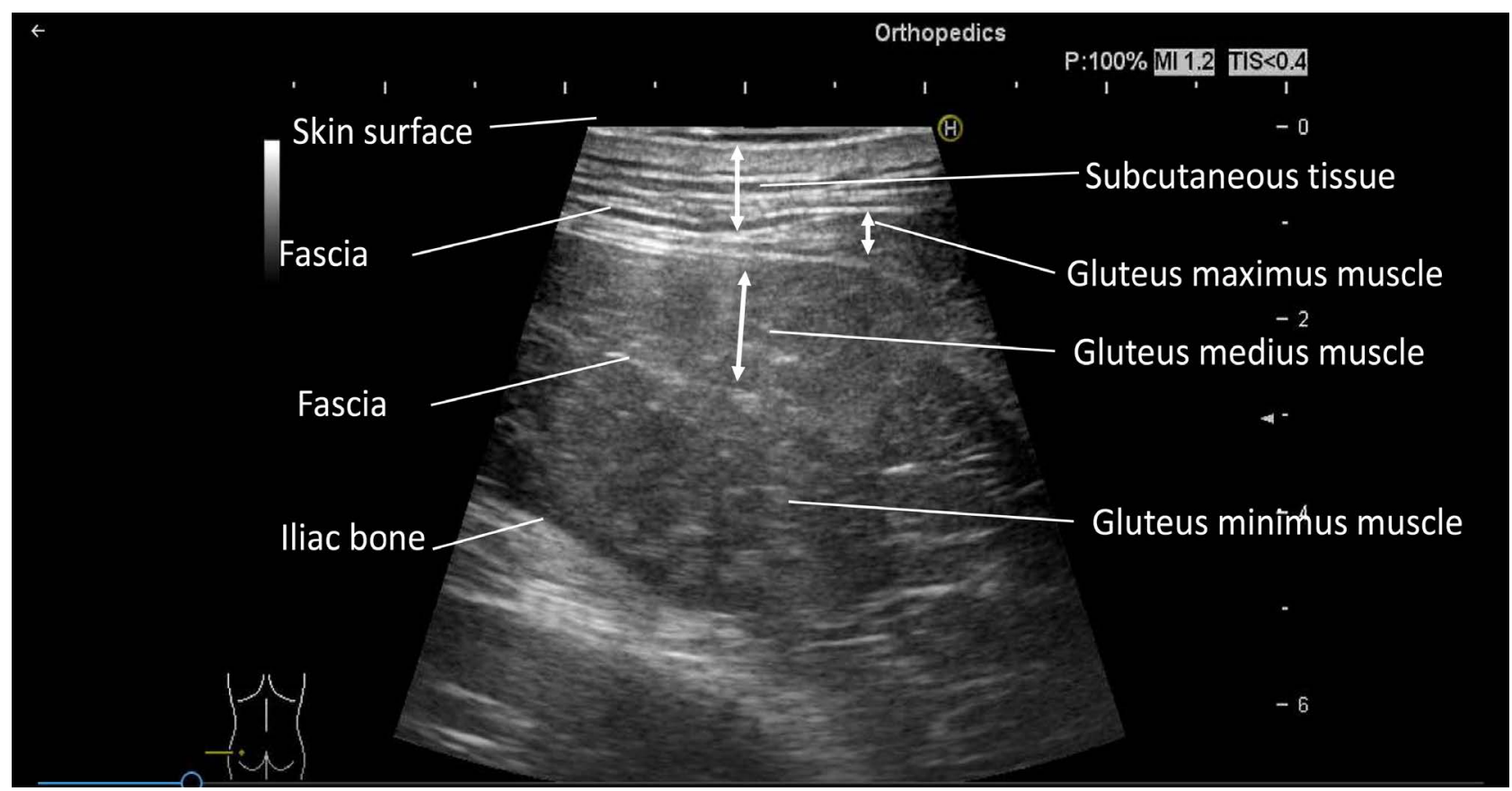

Figure 3. Ultrasonographic image at dorsoglutal site: Skin surface, subcutaneous tissue, fascia, gluteus muscles and ilium. The dorsogluteal site is exhibited with, the gluteus maximus muscle thinly covering the gluteus medius muscle. The distance from the epidermis to the fascia of the gluteus medius muscle (DEUF) is $15 \mathrm{~mm}$, and $50 \mathrm{~mm}$ from the epidermis to the bone (DEI).

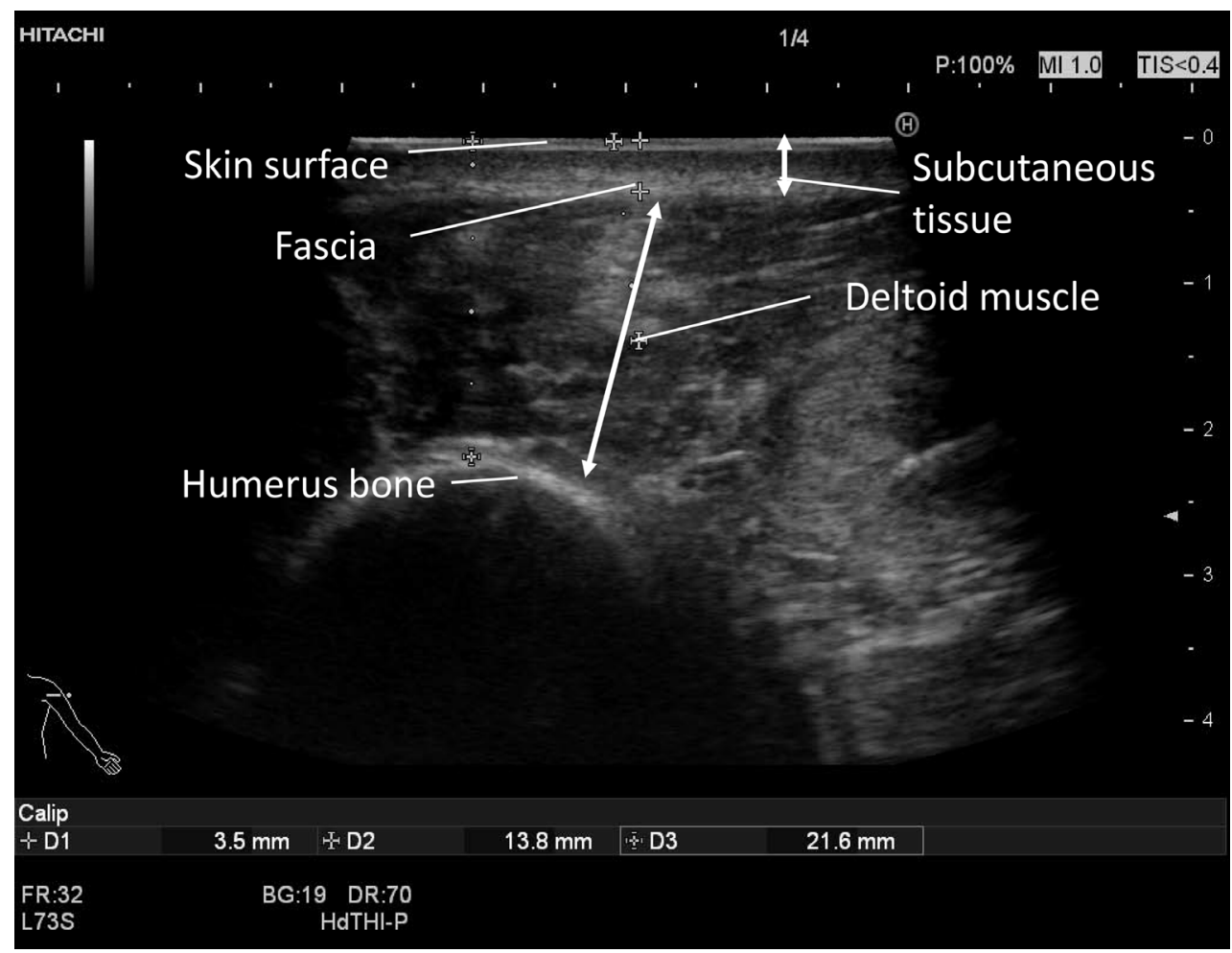

Figure 4. Ultrasonographic image of deltoid IM injection site (Right side): epidermis, subcutaneous tissue, muscle membrane, muscle and humerus. The measured site was the distance of "three finger-widths of the patient's finger (55 $\mathrm{mm})$ " below the acromion. The distance from the epidermis to the deltoid muscle fascia was $6.5 \mathrm{~mm}$, and the distance from the epidermis to the bone (DEI) was $21.6 \mathrm{~mm}$. 

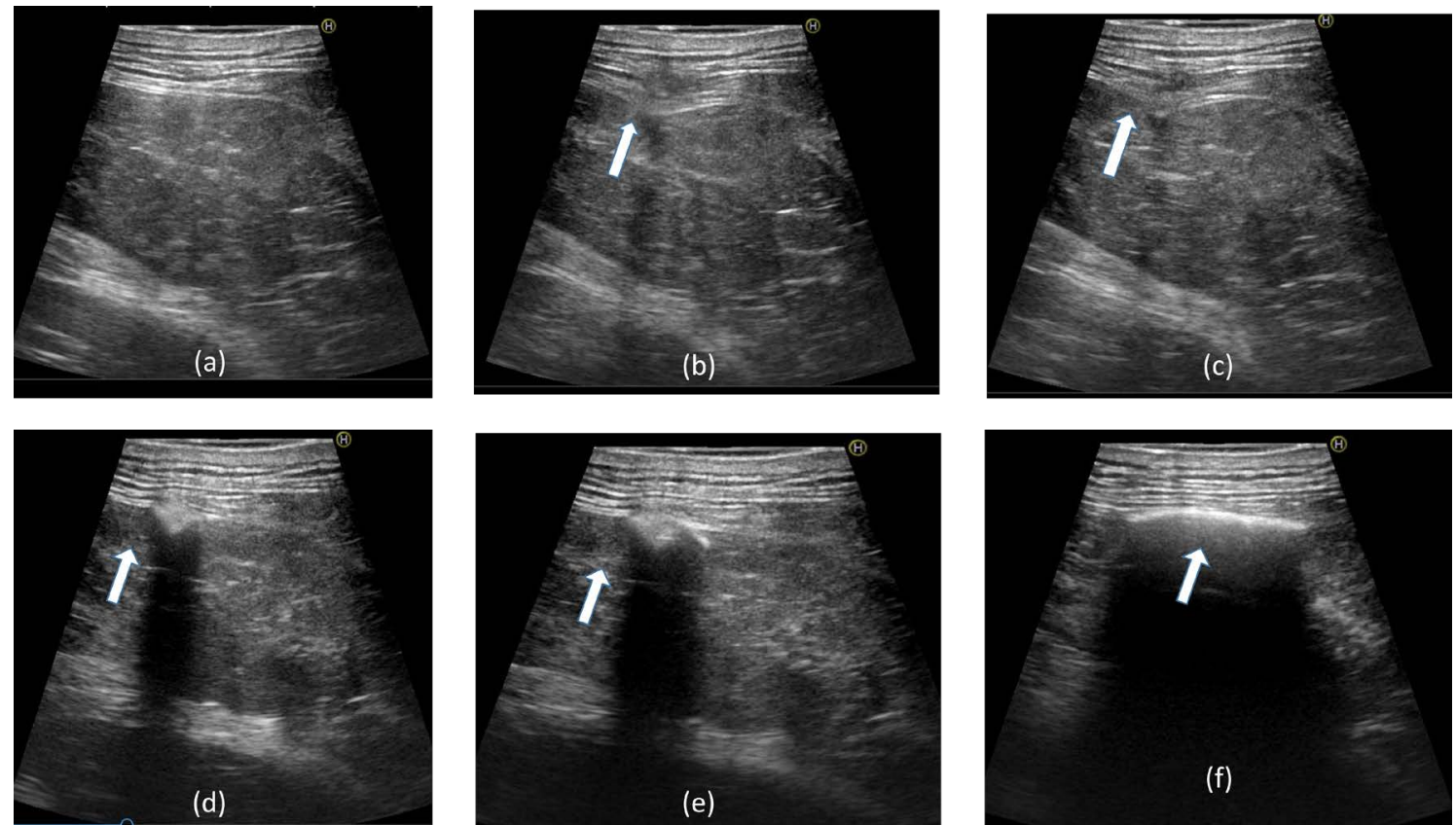

Figure 5. Aripiprazole long-acting injectable antipsychotics B-mode image (Left dorsogluteal site): injection needle during injection, fascia, muscle, injection solution, acoustic shadow. (a) Ultrasound image before IM injection: Before IM injection, the iliac bone appeared as an echogenic line. (b) View of gluteal muscle: By the insertion of the needle to the gluteus medius muscle, distortion of the fascia was observed. (c) The needle penetrated the fascia. (d) Image of RLAI at the initiation of the injection. Artifacts (acoustic shadow) due to the ALAI injection needle were observed. Image of ALAI injected into the gluteus medius, ALAI was observed as high echogenicity mass in the gluteus medius muscle. (e) Acoustic shadow is noted by the injection of ALAI, the iliac bone could not be verified in this image. (f) In this case, the injected ALAI was seen as high echogenicity line with marked acoustic shadowing. The ALAI was injected into the fascia of the gluteus maximus and medius.
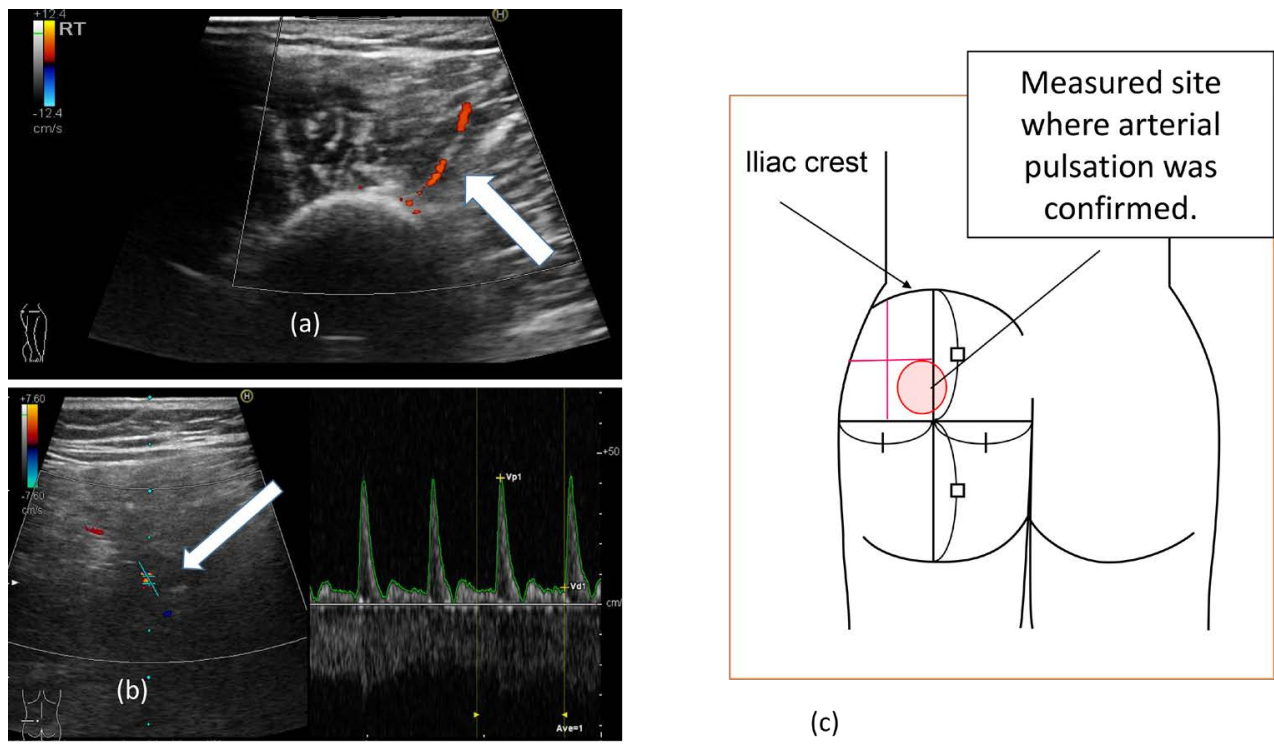

(c)

Figure 6. Detection of an artery by B-mode scan with colored Doppler image: (The deltoid site: top of the figure right side; the dorsoglutal site: bottom figure, left side) and buttocks measurement site. Figure 6 shows (a) a healthy male, the distance three finger widths from the acromion was the length of the patient's finger $(57 \mathrm{~mm})$. It was measured on the site. The deltoid site: Right-side, arterial pulsation confirmed. (b) Image of a schizophrenia patient. The dorsogluteal site: arterial pulsation confirmed within the gluteus medius muscle. (c) a diagram of the Doppler probe site, when the arterial pulsation was detected in the aforementioned patient. 
lumen separated by high echogenicity lines without Doppler signals may be nerves [41].

Surrounding the deltoid IM injection site, the thickness of the deltoid muscle, and especially the subcutaneous fat of the upper arm is thin compared to that of the buttocks. Care needs to be taken due to the numerous branches of the brachial plexus and brachial artery in the surrounding area.

\subsection{Evaluation of Induration at the IM Injection Site by Elastography}

The left side of Figure 7 is an image obtained by Elastography.

Elastography is an ultrasonic method for imaging the elasticity of compliant tissues [42]. By Elastography, the hardness of the tissue of the IM injection site can be evaluated, and the degree of the induration can be diagnosed. While the injection procedure performed incorrectly, especially in the cases with the needle was not inserted deeply enough to penetrate the fascia, the echogenic mass would be detected in the subcutaneous tissue on B-mode scan. In these cases, it could be a cause of focal induration and pain.

\subsection{Result of Comparison of DEUF and DEI of Four Classical Gluteal IM Injection Sites}

Figure 8 shows the comparison result of "Distance from the Epidermis to the Under-Fascia (DEUF)" and "Distance from the Epidermis to the Iliac Bone

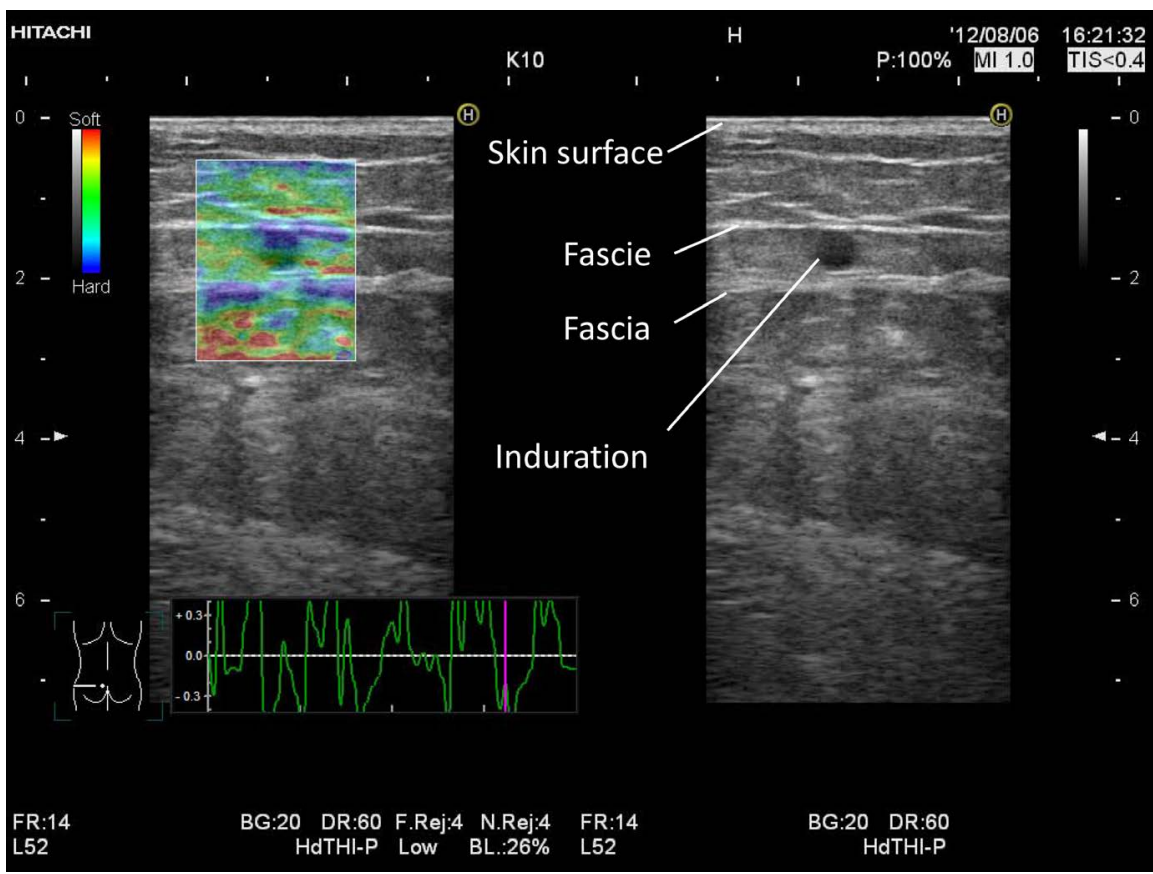

Figure 7. Induration confirmed by ultrasound diagnostic device: Woman with schizophrenia, 48 years, $163 \mathrm{~cm}, 80.6 \mathrm{~kg}$. The blue part indicates that the tissue is harder than the surroundings. B-mode image shows on the right. This subject had a history of treatment with first-generation anti-psychotic LAI Fluphenazine Decanoate $50 \mathrm{mg}$, oil-formulation. The condition of the drug post-LAI injection is able to be confirmed via ultrasonography. 


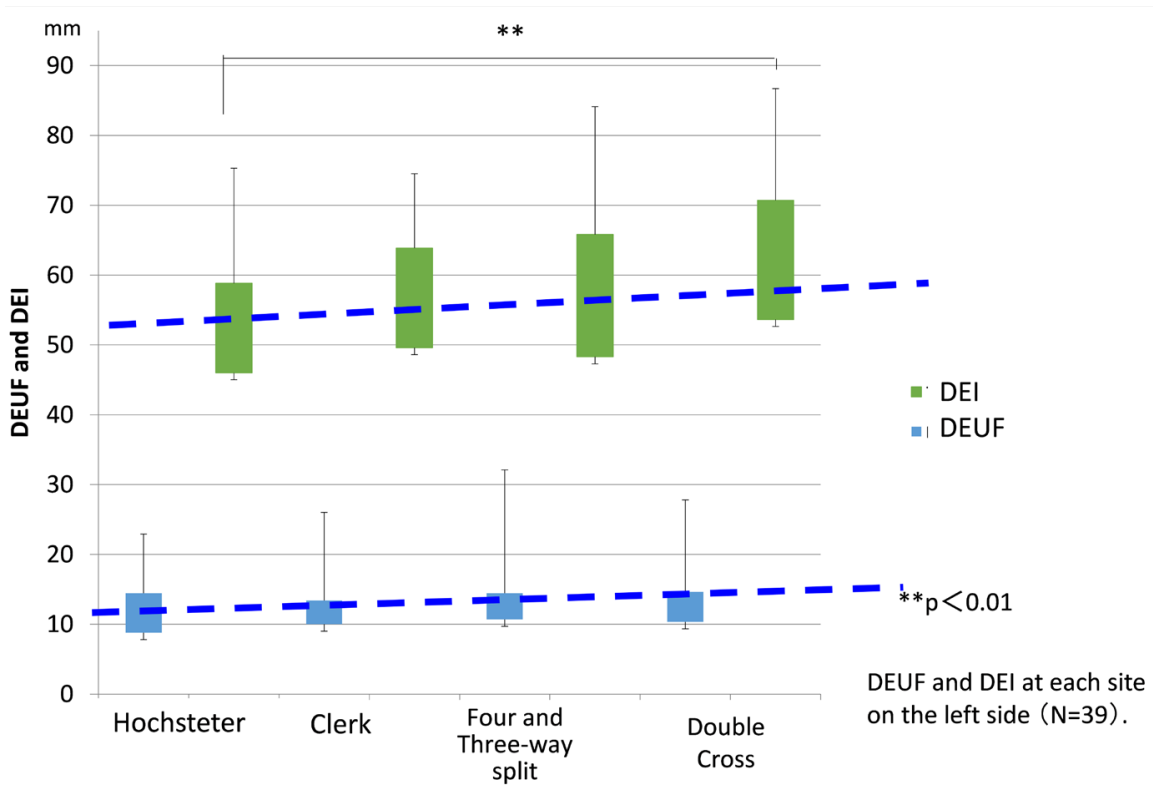

Figure 8. Comparison results of the four gluteal IM injection sites. The DEI became increasingly deeper in order of Hochstetter, Clark, Four and Three-way split and Double Cross. However, for the DEUF a significant difference between sites was not observed.

(DEI)" of the four classical gluteal IM injection sites. It is important to be mindful of the fact that DEI changes depending on injection site. In the interest of being concise, only the left side of the buttock is shown since there was almost no difference in sides. Please refer to the references for details [14].

\section{Points to Acquire IM Injection Technique}

\subsection{Preparation of IM Injection}

Before preparing for the procedure, it is necessary to verify, by enclosed package pamphlet and interview form, the necessity or not of massage, the properties of the medicine, meticulous, and usage. For medications which do not require massage, it is important to communicate this information to the patient for the purpose of ensuring optimum drug efficacy. Moreover, when carrying out repeated IM injections, the previously employed injection site and administered volume should be verified from the patient record, and the opposite side or alternate site selected for use.

\subsection{Selection of IM Injection Site}

When utilizing the gluteal IM injection sites, patient position at administration will differ depending on the site. Prone position is necessary for the four and three-way split method point and Double-cross site, while the Hochstetter site allows for lateral or standing position. In addition, both lateral and prone positions are possible for the Clerk site.

However, results from a study of females with average physique [30] indicated that for the Hochstetter site, skinfold thickness in supine position was $0.7 \mathrm{~cm}$ 
thicker than in lateral position. In consideration of this, skinfold thickness is thought to change depending on whether administering at the Hochstetter site in standing or lateral position.

When administering at the deltoid muscle, $5 \mathrm{~cm}$ below the acromion, slightly above the point three finger widths below the acromion, is considered to be safe in order to avoid damage to nerves or blood vessels [43]. It is important to use a tape measure and ensure that physicians and nurses understand the three-finger width measurement in order to select the most suitable point. Due to differences in height, weight and muscle volume between patients, the buttocks should be maintained as an option in cases where muscle volume is low or medication volume is high. Furthermore, it is important to verify the absence of swelling, redness and induration by palpation, as well as feelings of pain or discomfort by the patient.

\subsection{Notes When Administering Drugs}

Due to the proximity of the superior gluteal artery and nerve to the four and three-way split method and the double cross sites of the gluteus medius muscle, it is crucial that the absence of numbness, severe pain and blood in the syringe is confirmed before injecting the drug. Moreover, even after injection, it is important to check for presentation of delayed paralysis as part of the safety procedures for carrying out IM injections. As a rule, direction of the needle should be at 90 degrees to the skin in order to ensure the entire tip reaches the appropriate area.

When carrying out IM injections, the syringe should be held in the dominant hand, piercing perpendicular to the skin, using the pen-holding method as a means of keeping it steady.

Before injecting the medicine, it is crucial to verify with the patient that there is no numbness or severe pain. Pull back slightly on the plunger to check the absence of blood, if there is blood, withdraw the needle and stop the bleeding. When injecting the medicine, do not change hands, keep the syringe steady so as not to alter the direction or depth of the needle, use the opposite hand to deliver the medicine.

When administering IM injections at the deltoid injection site, it is important to maintain a posture where the syringe can be held steady, so as to ensure that the entire injection needle tip reaches the appropriate area. When physicians and nurses administer the injection needle by standing position, it is possible that the tip of the needle could enter the area proximal to the deltoid branch of the axillary nerve. To avoid risk factors such as nerve and blood vessel damage, and injecting a site different from that assessed, physicians and nurses should sit on a chair or something to administer the injection.

Physicians and nurses should always wear gloves to comply with standard precautions against infection.

Factors influencing IM injection administration: 
1) Needle-based factors: kinds of syringes, injection needles, drugs.

2) Factors about the patients: Optimum constant posture can be obtained, selection and condition of injection site; a) Condition such as physique, subcutaneous fat, muscle mass, skin, b) Neurological factors: the nerve conditions requiring attention, blood circulation surrounding the blood vessels (in the vicinity of the injection penetration site), and c) Subcutaneous tissue and muscular condition at the injection site by injecting liquid medicine as previously noticed.

3) Knowledge shared by physicians and nurses need to be enforced: Knowledge of the anatomical positions of skin and muscles, and the nerve and vessel circulatory health require attention, enforcing muscle injection guidelines (penetration point, depth, direction), and urgent judgment at the time of implementation of procedures.

\section{Conclusions}

It is essential to deliver the drug accurately into the muscle in order to achieve adequate drug efficacy and prevent injection site reactions from IM injections. Therefore, when delivering an IM injection of a drug without an attached specialist needle to the buttocks, a suitable injection needle between $23 \mathrm{G} 25 \mathrm{~mm}$ to $21 \mathrm{G}$ or $22 \mathrm{G} 38 \mathrm{~mm}$, must be chosen depending on physique and particular drug characteristics. In the case of a deltoid injection site, a 23G $25 \mathrm{~mm}$ injection needle is used in the absence of notable obesity. It is important to stretch the skin, penetrating at 90 degrees, and to ensure that the needle reaches the muscle.

Given the risk of nerve or arteriovenous injury and depth of the injection, not good to say the dorsogluteal site is preferable to the ventrogluteal site. However, the dorsogluteal site is used more in clinical practice [44]. Also, the risk of nerve and artery injury in the deltoid injection site is higher than in the buttocks. It is necessary to be careful when applying a deltoid injection. Until new confirmed evidence becomes available in the future, it is necessary to make an effort to evaluate the methods of muscular injection, such as injection site selection methods, needle penetration depth, needle angle, and established IM injection techniques. The developments of the ultrasound devices may promise the next-generation innovative techniques for IM injection. Importantly, these techniques provide critical information from aspect of a risk management.

\section{Funding/Financial Disclosure}

This work was supported by TERUMO FOUNDATION for LIFE SCIENCE and ARTS.

\section{References}

[1] Brissos, S., Veguilla, M.R., Taylor, D. and Balanzá-Martinez, V. (2014) The Role of Long-Acting Injectable Antipsychotics in Schizophrenia: A Critical Appraisal Psychopharmacology. Therapeutic Advances in Psychopharmacology, 4, 198-219. https://doi.org/10.1177/2045125314540297

[2] Choktanasiri, W., Boonkasemsanti, W., Sittisomwong, T., Kunathikom, S., Suk- 
sompong, S., Udomsubpayakul, U. and Rojanasakul, A. (1996) Long-Acting Triptorelin for the Treatment of Endometriosis. International Journal of Gynecology \& Obstetrics, 54, 237-243. https://doi.org/10.1016/0020-7292(96)02698-7

[3] Kaunitz, A.M. (1998) Injectable Depot Medroxyprogesterone Acetate Contraception: An Update for U.S. Clinicians. International Journal of Fertility and Women's Medicine, 43, 73-83.

[4] Ravenstijn, P., Remmerie, B., Savitz, A., Samtani, M.N., Nuamah, I., Chang, C.T., De Meulder, M., Hough, D. and Gopal, S. (2016) Pharmacokinetics, Safety, and Tolerability of Paliperidone Palmitate 3-Month Formulation in Patients with Schizophrenia: A Phase-1, Single-Dose, Randomized, Open-Label Study. The Journal of Clinical Pharmacology, 56, 330-339. https://doi.org/10.1002/jcph.597

[5] Takada, S. and Kawanishi, C. (2006) Injection Skills Based upon Evidence. Nakayama Shoten Co. Ltd., Tokyo, 123-128. (In Japanese)

[6] Hamann, G.L., Egan, T.M., Wells, B.G. and Grimmig, J.E. (1990) Injection Site Reactions after Intramuscular Administration of Haloperidol Decanoate 100 $\mathrm{mg} / \mathrm{mL}$. The Journal of Clinical Psychiatry, 51, 502-504.

[7] Duque, F.L.V. and Chagas, C.A.A. (2009). Intramuscular Accident with Drug Injection in the Deltoid Muscle: Local and Distant Lesions, Review of 32 Cases. Jornal Vascular Brasileiro, 8, 238-246. https://doi.org/10.1590/S1677-54492009000300009

[8] Mishara, P. and Stringer, M.D. (2010) Sciatic Nerve Injury from Intramuscular Injection: A Persistent and Global Problem. The International of Journal of Clinical Practice, 64, 1573-1579. https://doi.org/10.1111/j.1742-1241.2009.02177.x

[9] Cook, I.F. (2011) An Evidence Based Protocol for the Prevention of Upper Arm Injury Related to Vaccine Administration (UAIRVA). Human Vaccines, 7, 845-848. https://doi.org/10.4161/hv.7.8.16271

[10] Jones, J.C., Day, J.C., Taylor, J.R. and Thomas, C.S. (1998) Investigation of Depot Neuroleptic Injection Site Reactions. Psychiatry Bulletin, 22, 605-607. https://doi.org/10.1192/pb.22.10.605

[11] Zhao, Y., Tanioka, T., Yasuhara, Y., Takase, K., Tsuboi, S., Fujita, K., Locsin, R. and Iwata, N. (2017) Effects of Depth of Needle Insertion with Risperidone Long-Acting Injectable in Persons with Schizophrenia: A Randomized Double-Blind Study. Open Journal of Psychiatry, 7, 374-385. https://doi.org/10.4236/ojpsych.2017.74031

[12] Hara, Y. and Shinozaki, E. (2017) Research on Clinical Nurses' Intramuscular Injection Techniques and the Induration Site with Intramuscular Injection of an Oil-Based Solution. Japanese Journal of Nursing Art and Science, 16, 51-60. (In Japanese)

[13] Palma, S. and Strohfus, P. (2013) Are IM Injections IM in Obese and Overweight Females? A Study in Injection Technique. Applied Nursing Research, 26, e1-e4. https://doi.org/10.1016/j.apnr.2013.09.002

[14] Masuda, S., Tanioka, T., Yasuhara, Y., Tanioka, T., Atsuta, A., Motoki, K., Takase, K. and Locsin, R. (2016) Comparison of Gluteal Muscle Intramuscular Injection Sites of Japanese Healthy Subjects: Considerations for Optimal Insertion of Injection Needle Length. Open Journal of psychiatry, 6, 203-212. https://doi.org/10.4236/ojpsych.2016.62025

[15] Sakamaki, S., Yasuhara, Y., Motoki, K., Takase, K., Tanioka, T. and Locsin, R. (2013) The Relationship between Body Mass Index, Thickness of Subcutaneous Fat, and the Gluteus Muscle as the Intramuscular Injection Site. Health, 5, 1443-1448. https://doi.org/10.4236/health.2013.59196 
[16] Wynadena, D., Tohotoa, J., Omari, O.A.L., Happell, B., Heslop, K., Barrd, L. and Sourinathand, V. (2015) Administering Intramuscular Injections: How Does Research Translate into Practice over Time in the Mental Health Setting? Nurse Education Today, 35, 620-624. http://dx.doi.org/10.1016/j.nedt.2014.12.008

[17] Yasuhara, Y., Tanioka, T. Takase, K., Zhao, Y., Motoki, K. and Azekawa, T. (2017) Evaluations of Muscle Echogenicities in Patients Treated with Second Generation Long Acting Injectable Antipsychotics. Neurosonology, 30, 13-17.

[18] Tanioka, T., Sakamaki, S., Yasuhara, Y., Tomotake, M., Takase, K., Watari, C., Makiguchi, K., Locsin, R., Motoki, K. and Inui, T. (2013) Optimal Needle Insertion Length for Intramuscular Injection of Risperidone Long-Acting Injectable (RLAI). Health, 5, 1939-1945. https://doi.org/10.4236/health.2013.512262

[19] Yasuhara, Y., Hirai, E., Sakamaki, S., Tanioka, T., Motoki, K., Takase, K., Locsin, R., Kawanishi, C., Inui, T., Watari, C. and Makiguchi, K. (2012) Using Ultrasonography in Evaluating the Intramuscular Injection Techniques Used for Administering Drug Treatments to Schizophrenic Patients in Japan. The Journal of Medical Investigation, 59, 213-219.

[20] Masuda, S., Sakamaki, S., Yasuhara, Y., Zhao, Y., Takase, K., Kai, Y., Tanioka, T. and Locsin, R. (2016) Explanatory Variables as Evidence for Precision in Intramuscular Long-Acting Injections of Patients with Schizophrenia. Open Journal of Psychiatry, 6, 125-134. https://doi.org/10.4236/ojpsych.2016.61015

[21] Tanioka, T. and Yasuhara, Y., Editorial Supervision (2016) Pocket Guide; Points for Intramuscular Injection Long Acting Injectables (LAI). Janssen Pharma Co., Ltd., Tokyo. (In Japanese)

[22] Iwanaga, H. and Takayama, S. (2003) Study of the Suitable Sites for the Intramuscular Injection into the Deltoid Muscle or the Gluteus Medius Muscle. Tokai University, School of Health Sciences Bulletin, 9, 29-33. (In Japanese)

[23] Pandian, J.D., Bose, S., Daniel, V., Singh, Y. and Abraham, A.P. (2006) Nerve Injuries Following Intramuscular Injections: A Clinical and Neurophysiological Study from Northwest India. Journal of the Peripheral Nervous System, 11, 165-171. https://doi.org/10.1111/j.1085-9489.2006.00082.x

[24] Cocoman, A. and Murray, J. (2008) Intramuscular Injections: A Review of Best Practice for Mental Health Nurses. Journal of Psychiatric and Mental Health Nursing, 15, 424-434. https://doi.org/10.1111/j.1365-2850.2007.01236.x

[25] Small, S.P. (2004) Preventing Sciatic Nerve Injury from Intramuscular Injections: Literature Review. Journal of Advanced Nursing, 47, 287-296. https://doi.org/10.1111/j.1365-2648.2004.03092.x

[26] Cocoman, A. and Murray, J. (2010) Recognizing the Evidence and Changing Practice on Injection Sites. British Journal of Nursing, 19, 1170-1174. https://doi.org/10.12968/bjon.2010.19.18.79050

[27] Nicoll, L.H. and Hesby A. (2002) Intramuscular Injection: An Integrative Research Review and Guideline for Evidence-Based Practice. Applied Nursing Research, 15, 1490-1162. https://doi.org/10.1053/apnr.2002.34142

[28] Coskun, H., Kilic, C. and Senture, C. (2016) The Evaluation of Dorsogluteal and Ventrogluteal Injection Sites: A Cadaver Study. Journal of Clinical Nursing, 25, 1112-1119. https://doi.org/10.1111/jocn.13171

[29] Bodor, M. and Montalvo, E. (2007) Vaccination-Related Shoulder Dysfunction. Vaccine, 25, 585-587. https://doi.org/10.1016/j.vaccine.2006.08.034

[30] Takahashi, Y., Kikuchi, K., Miura, N. and Ishida, Y. (2014) Appropriate Needle Insertion Depth for Intramuscular Injection Based on Assessment of BMI. Journal of 
Japan Academy of Nursing Science, 34, 36-45. (In Japanese)

[31] Nakajima, Y., Mukai, K., Takaoka, K., Hirose, T., Morishita, K., Yamamoto, T., Yoshida, Y., Urai, Y. and Nakatani, T. (2017) Establishing a New Appropriate Intramuscular Injection Site in the Deltoid Muscle. Human Vaccines \& Immunotherapeutics, 13, 2123-2129. https://doi.org/10.1080/21645515.2017.1334747

[32] Haramati, N., Lorans, R., Lutwin, M. and Kaleya, R.N. (1994) Injection Granulomas. Intramuscle or Intrafat? Archives of Family Medicine, 3, 146-148. https://doi.org/10.1001/archfami.3.2.146

[33] Kikuchi, K., Takahashi, Y., Oyama, N. and Ishida, Y. (2009) Needle Length Required for Intramuscular Injection Skills: Evidence-Based Nursing. Japanese Journal of Nursing Art and Science, 8, 66-75. (In Japanese)

[34] Sato, Y., Narita, S. and Nakano, T. (2005) A Study on the Method of Choosing Intramuscular Injection Site in the Buttocks. Japanese Journal of Nursing Research, 28, 45-52. (In Japanese)

[35] Takahashi, Y., Kikuchi, K., Oyama, N., Yoko, I. and Sato, F. (2007) Investigation about the Intramuscular Injection Method Prevent Medication from Leaking Back onto the Skin in Psychiatry. Journal of the Faculty of Nursing, Iwate Prefectural University, 9, 103-112. (In Japanese)

[36] Chan, V.O., Colville, J., Persaud, T., Buckley, O., Hamilton, S. and Torreggiani, W.C. (2006) Intramuscular Injections into the Buttocks: Are They Truly Intramuscular? European Journal of Radiology, 58, 480-484.

https://doi.org/10.1016/j.ejrad.2006.01.008

[37] Yasuhara, Y., Sakamaki, S., Tanioka, T., Motoki, K., Sasakawa, C., Takase, K. and Kawanishi, C. (2013) Optimal Length of Intramuscular Injection Needle and Drug Absorption by Ultrasound Evaluation. The Japan Academy of Neurosonology, 25, 91-94.

[38] Gopal, S., Gassmann-Mayer, C., Palumbo, J., Samtani, M.N., Shiwach, R. and Alphs, L. (2010) Practical Guidance for Dosing and Switching Paliperidone Palmitate Treatment in Patients with Schizophrenia. Current Medical Research and Opinion, 26, 377-387. https://doi.org/10.1185/03007990903482772

[39] Sugawara, N., Sagae, T., Yasui-Furukori, N., Yamazaki, M., Shimoda, K., Mori, T., Sugai, T., Matsuda, H., Suzuki, Y., Ozeki, Y., Okamoto, K. and Someya, T. (2018) Effects of Nutritional Education on Weight Change and Metabolic Abnormalities among Patients with Schizophrenia in Japan: A Randomized Controlled Trial. Journal of Psychiatric Research, 97, 77-83. https://doi.org/10.1016/j.jpsychires.2017.12.002

[40] Suzuki, Y., Sugai, T., Fukui, N., Watanabe, J., Ono, S., Tsuneyama, N., Saito, M. and Someya, T. (2014) High Prevalence of Underweight and Undernutrition in Japanese Inpatients with Schizophrenia. Psychiatry and Clinical Neurosciences, 68, 78-82. https://doi.org/10.1111/pcn.12082

[41] Sakamaki, S., Zhao, Y., Yasuhara, Y., Motoki, K., Takase, K., Abe, H., Miyazaki, K. and Tanioka, T. (2014) Consideration of Long Acting Injection Procedure into the Gluteal Medius Muscle by Real-Time B-Mode Ultrasonography: Case Reports of Patients Who Observed Injection Site Reactions or Blood Back-Flow. Japanese Journal of Clinical Psychopharmacology, 17, 253-260. (In Japanese)

[42] Céspedes, I., Ophir, J., Ponnekanti, H. and Maklad, N. (1993) Elastography: Elasticity Imaging Using Ultrasound with Application to Muscle and Breast in Vivo. UItrasonic Imaging, 15, 73-88. https://doi.org/10.1177/016173469301500201

[43] Kikuchi, K., Oyama, N., Takahashi, Y. and Ishida, Y. (2009) Analysis of Practical 
Knowledge of Deltoid Intramuscular Injections by Nurses in Hospitals. Journal of the Faculty of Nursing, Iwate Prefectural University, 11, 79-85. (In Japanese)

[44] Tuck, S.O. (2014) Intramuscular Injection Technique: An Evidence-Based Approach. CPD (Continuing Professional Development), 29, 52-59.

https://rcni.com/sites/rcn_nspace/files/ns.29.4.52.e9183.pdf 